\title{
Publisher's Note: Hybrid functionals with fixed mixing parameter perform no better than PBE for fundamental band gaps of nanoscale materials [Phys. Rev. B 94, 195429 (2016)]
}

Xinquan Wang, Marc Dvorak, and Zhigang Wu

(Received 2 December 2016; published 13 December 2016)

DOI: 10.1103/PhysRevB.94.239904

This paper was published online on 21 November 2016 with a typographical error in the author list. The second author's name should read as "Marc Dvorak". The author name has been corrected as of 2 December 2016. The author name is correct in the printed version of the journal. 